\title{
Semileptonic $B$-meson decays to light pseudoscalar mesons on the HISQ ensembles
}

\author{
Zechariah Gelzer ${ }^{1,2, \star}$, C. Bernard ${ }^{3}$, C. DeTar ${ }^{4}$, A.X. El-Khadra ${ }^{1,5}$, E. Gámiz ${ }^{6}$, Steven Gottlieb ${ }^{7}$, \\ Andreas S. Kronfeld ${ }^{5,8, \star \star}$, Yuzhi Liu ${ }^{7}$, Y. Meurice ${ }^{2}$, J.N. Simone ${ }^{5}$, D. Toussaint ${ }^{9}$, R.S. Van de \\ Water ${ }^{5}$, and R. Zhou ${ }^{5}$ \\ ${ }^{1}$ Department of Physics, University of Illinois, Urbana, IL 61801, USA \\ ${ }^{2}$ Department of Physics and Astronomy, University of lowa, lowa City, IA 52242, USA \\ ${ }^{3}$ Department of Physics, Washington University, St. Louis, Missouri, 63130, USA \\ ${ }^{4}$ Department of Physics and Astronomy, University of Utah, Salt Lake City, Utah 84112, USA \\ ${ }^{5}$ Fermi National Accelerator Laboratory, Batavia, Illinois, USA \\ ${ }^{6}$ CAFPE and Departamento de Física Teórica y del Cosmos, Universidad de Granada, Granada, Spain \\ ${ }^{7}$ Department of Physics, Indiana University, Bloomington, Indiana 47405, USA \\ ${ }^{8}$ Institute for Advanced Study, Technische Universität München, D-85748 Garching, Germany \\ ${ }^{9}$ Department of Physics, University of Arizona, Tucson, Arizona 85721, USA
}

\begin{abstract}
We report the status of an ongoing lattice-QCD calculation of form factors for exclusive semileptonic decays of $B$ mesons with both charged currents $(B \rightarrow \pi \ell v$, $\left.B_{s} \rightarrow K \ell v\right)$ and neutral currents $\left(B \rightarrow \pi \ell^{+} \ell^{-}, B \rightarrow K \ell^{+} \ell^{-}\right)$. The results are important for constraining or revealing physics beyond the Standard Model. This work uses MILC's $(2+1+1)$-flavor ensembles with the HISQ action for the sea and light valence quarks and the clover action in the Fermilab interpretation for the $b$ quark. Simulations are carried out at three lattice spacings down to $0.088 \mathrm{fm}$, with both physical and unphysical seaquark masses. We present preliminary results for correlation-function fits.
\end{abstract}

\section{Introduction}

Semileptonic decays of $B_{(s)}$ mesons are important to searches for new physics at the intensity frontier. Decays mediated at tree-level by charged currents (see Figure 1, left), namely $B \rightarrow \pi \ell v_{\ell}$ and $B_{s} \rightarrow K \ell v_{\ell}$, provide precise determinations of the CKM matrix element $\left|V_{u b}\right|$. This element is crucial for testing CKM unitarity and thus important in the search for new physics. There are well-known discrepancies between inclusive and exclusive determinations of $\left|V_{u b}\right|$ [1]. Exclusive determinations combine theoretical calculations of nonperturbative form factors with experimental measurements of decay rates. This work seeks to further reduce the uncertainties from theory.

Decays mediated by neutral currents (see Figure 1, right), namely $B \rightarrow \pi \ell^{+} \ell^{-}$and $B \rightarrow K \ell^{+} \ell^{-}$, provide precise tests of the Standard Model. Since flavor-changing neutral-current (FCNC) interactions are rare, they are sensitive to contributions from new physics. Tension currently exists between

\footnotetext{
$\star$ e-mail: zgelzer@illinois.edu

${ }^{\star}$ Speaker, e-mail: ask@fnal.gov
} 

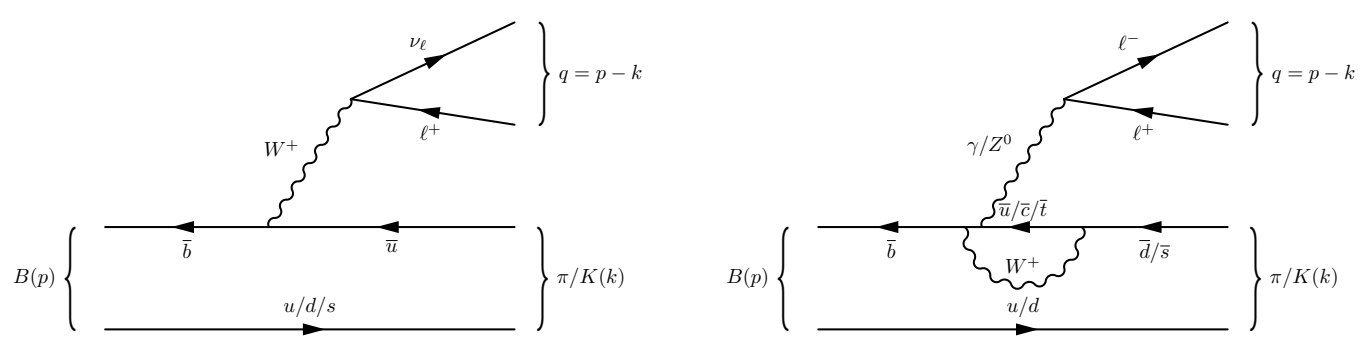

Figure 1. Feynman diagrams for tree-level charged-current decays $B_{(s)} \rightarrow \pi(K) \ell v_{\ell}$ (left) and rare flavor-changing neutral-current interactions $B \rightarrow \pi(K) \ell^{+} \ell^{-}$(right), with all QCD corrections suppressed.

the Standard Model and experimental data pertaining to these FCNC interactions [2]. The semileptonic decays and neutral mixing of $B$ mesons have been studied in experiments at BABAR [3], Belle [4], CDF [5], DØ [6], and LHCb [7-9] and will become available from ongoing measurements at $\mathrm{LHCb}$ and the upcoming Belle II experiment. Errors from theory and experiment are commensurate and expected to be improved.

In this work, we calculate form factors from first principles in lattice QCD for the exclusive decays $B \rightarrow \pi \ell v_{\ell}, B_{s} \rightarrow K \ell v_{\ell}, B \rightarrow \pi \ell^{+} \ell^{-}$, and $B \rightarrow K \ell^{+} \ell^{-}$. We use (2+1+1)-flavor MILC HISQ ensembles, where the light quarks are at physical masses. The purpose of this work is to improve theoretical confrontations with experiment, both by determining $\left|V_{u b}\right|$ in charged-current decays and by comparing branching fractions in neutral-current decays.

\section{Matrix elements and form factors}

In the Standard Model and beyond, transitions between pseudoscalar mesons can be mediated by a vector current $\mathcal{V}^{\mu}=\bar{q} \gamma^{\mu} b$, a tensor current $\mathcal{T}^{\mu \nu}=i \bar{q} \sigma^{\mu \nu} b$, or a scalar current $\mathcal{S}=\bar{q} b$. The resulting matrix elements are as follows:

$$
\begin{aligned}
\left\langle P(k)\left|\mathcal{V}^{\mu}\right| B(p)\right\rangle & =f_{+}\left(q^{2}\right)\left(p^{\mu}+k^{\mu}-\frac{M_{B}^{2}-M_{P}^{2}}{q^{2}} q^{\mu}\right)+f_{0}\left(q^{2}\right) \frac{M_{B}^{2}-M_{P}^{2}}{q^{2}} q^{\mu}, \\
& =\sqrt{2 M_{B}}\left[v^{\mu} f_{\|}\left(E_{P}\right)+k_{\perp}^{\mu} f_{\perp}\left(E_{P}\right)\right], \\
\left\langle P(k)\left|\mathcal{T}^{\mu v}\right| B(p)\right\rangle & =f_{T}\left(q^{2}\right) \frac{2}{M_{B}+M_{P}}\left(p^{\mu} k^{v}-p^{v} k^{\mu}\right), \\
\langle P(k)|\mathcal{S}| B(p)\rangle & =f_{0}\left(q^{2}\right) \frac{M_{B}^{2}-M_{P}^{2}}{m_{b}-m_{q}}
\end{aligned}
$$

where $P=\pi, K$ is the light-pseudoscalar meson in the final state and $k$ its four-momentum, $B=B, B_{s}$ is the $B_{(s)}$ meson in the initial state and $p$ its four-momentum, and $q=p-k$ is the four-momentum carried off by the leptons. In Eq. (2.2), $f_{+}$and $f_{0}$ are rewritten in terms of $f_{\perp}$ and $f_{\|}$, where $v=p / M_{B}$ is the four-velocity of the $B$ meson, $k_{\perp}=k-(p \cdot v) v$ is the projection of the four-momentum of the final-state meson in the direction perpendicular to $v$, and $E_{P}=k \cdot v$ is the energy of the final-state meson in the $B$-meson rest frame. Thus we may reconstruct $f_{+}\left(q^{2}\right)$ and $f_{0}\left(q^{2}\right)$ as linear combinations of $f_{\perp}\left(E_{P}\right)$ and $f_{\|}\left(E_{P}\right)$ : 
Table 1. Parameter inputs and elementary outputs used in this work. Columns are (from left to right): approximate lattice spacing, lattice size, quark masses in lattice units, $b$-quark hopping parameter, number of configurations and different source times, product of pion mass with linear spatial dimension, and gradient-flow quantity.

\begin{tabular}{cccccccccc}
\hline$\approx a(\mathrm{fm})$ & $N_{s}^{3} \times N_{t}$ & $a m_{l}^{\prime}$ & $a m_{s}^{\prime}$ & $a m_{c}^{\prime}$ & $\kappa_{b}^{\prime}$ & $N_{\text {cfg }} \times N_{\text {src }}$ & $T$ & $a M_{\pi} N_{s}$ & $w_{0} / a$ \\
\hline 0.15 & $32^{3} \times 48$ & 0.00235 & 0.0647 & 0.831 & 0.07732 & $3630 \times 8$ & 14 & 3.3 & $1.1468(4)$ \\
\hline 0.12 & $24^{3} \times 64$ & 0.0102 & 0.0509 & 0.635 & 0.08574 & $1053 \times 8$ & 17 & 4.5 & $1.3835(10)$ \\
0.12 & $32^{3} \times 64$ & 0.00507 & 0.0507 & 0.628 & 0.08574 & $1000 \times 8$ & 17 & 4.3 & $1.4047(9)$ \\
0.12 & $48^{3} \times 64$ & 0.00184 & 0.0507 & 0.628 & 0.08574 & $986 \times 8$ & 17 & 4.0 & $1.4168(10)$ \\
\hline 0.088 & $64^{3} \times 96$ & 0.0012 & 0.0363 & 0.432 & 0.09569 & $925 \times 8$ & 25 & 3.7 & $1.9470(13)$ \\
\hline
\end{tabular}

$$
\begin{aligned}
f_{+}\left(q^{2}\right) & =\frac{1}{\sqrt{2 M_{B}}}\left[f_{\|}\left(E_{P}\right)+\left(M_{B}-E_{P}\right) f_{\perp}\left(E_{P}\right)\right] \\
f_{0}\left(q^{2}\right) & =\frac{\sqrt{2 M_{B}}}{M_{B}^{2}-M_{P}^{2}}\left[\left(M_{B}-E_{P}\right) f_{\|}\left(E_{P}\right)+\left(E_{P}^{2}-M_{P}^{2}\right) f_{\perp}\left(E_{P}\right)\right] .
\end{aligned}
$$

Eqs. (2.5) and (2.6) automatically satisfy the kinematic constraint $f_{+}(0)=f_{0}(0)$. It becomes straightforward to obtain the form factors $f_{\perp}, f_{\|}$, and $f_{T}$ as functions of the pion or kaon energy by analyzing two- and three-point correlation functions in the lattice-QCD calculation.

\section{Lattice-QCD calculation}

We use $(2+1+1)$-flavor ensembles generated by the MILC Collaboration, which include the dynamical effects of $u, d, s$, and $c$ quarks in the sea. The isospin limit $m_{u}=m_{d}=m_{l}$ is used for the light quarks and the same input masses are used for corresponding sea and valence quarks. There are five ensembles at three lattice spacings down to $0.088 \mathrm{fm}$ and three of these ensembles use physical quark masses. The highly-improved staggered quark (HISQ) action is used for the sea and light valence quarks, while the clover action in the Fermilab interpretation is used for the $b$ quark. Gluons are implemented with the one-loop improved Lüscher-Weisz action. To determine the lattice scale, we use the gradient-flow quantity $w_{0} / a$ [10], whose continuum value is given by $w_{0}=0.1714(15) \mathrm{fm}[11]$. Ensemble properties, including both parameter inputs and elementary outputs, are given in Table 1.

The lattice-QCD calculations in this work employ two- and three-point correlation functions defined as follows:

$$
\begin{aligned}
C_{2}^{B}(t ; \mathbf{0}) & =\sum_{\boldsymbol{x}}\left\langle O_{B}(t, \boldsymbol{x}) O_{B}^{\dagger}(0, \mathbf{0})\right\rangle, \\
C_{2}^{P}(t ; \boldsymbol{k}) & =\sum_{\boldsymbol{x}}\left\langle O_{P}(t, \boldsymbol{x}) O_{P}^{\dagger}(0, \mathbf{0})\right\rangle e^{-i \boldsymbol{k} \cdot \boldsymbol{x}}, \\
C_{3}^{\mu(v)}(t, T ; \boldsymbol{k}) & =\sum_{x, \boldsymbol{y}} e^{i \boldsymbol{k} \cdot \boldsymbol{y}}\left\langle O_{P}(0, \mathbf{0}) J^{\mu(v)}(t, \boldsymbol{y}) O_{B}^{\dagger}(T, \boldsymbol{x})\right\rangle,
\end{aligned}
$$

where $J^{\mu(v)}=V^{\mu}, T^{\mu v}$ are the lattice currents. The final-state-meson momenta are generated up to $\boldsymbol{k}=(4,0,0) \times 2 \pi /\left(a N_{s}\right)$. Correlation functions are computed from multiple source locations on each configuration to increase statistics. This is accomplished by setting eight temporal source locations 
Table 2. Fit ranges $\left[t_{\min }, t_{\max }\right]$ for each meson in fits to two-point correlators, where $t_{\max }$ is quoted at $\boldsymbol{k}=\mathbf{0}$.

\begin{tabular}{cccccc}
\hline$\approx a(\mathrm{fm})$ & $a m_{l}^{\prime} / a m_{s}^{\prime}$ & $B$ & $B_{s}$ & $K$ & $\pi$ \\
\hline 0.15 & $0.00235 / 0.0647$ & {$[2,15]$} & {$[2,24]$} & {$[4,23]$} & {$[4,23]$} \\
\hline 0.12 & $0.0102 / 0.0509$ & {$[3,18]$} & {$[3,24]$} & {$[5,31]$} & {$[5,31]$} \\
0.12 & $0.00507 / 0.0507$ & {$[3,17]$} & {$[3,26]$} & {$[5,31]$} & {$[5,31]$} \\
0.12 & $0.00184 / 0.0507$ & {$[3,17]$} & {$[3,29]$} & {$[5,31]$} & {$[5,31]$} \\
\hline 0.088 & $0.0012 / 0.0363$ & {$[4,23]$} & {$[4,41]$} & {$[7,47]$} & {$[7,47]$} \\
\hline
\end{tabular}

at $t=0, N_{t} / 8, \ldots, 7 N_{t} / 8$, while fixing the spatial source locations at $\boldsymbol{x}=\mathbf{0}$. The autocorrelations between successive configurations are mitigated by binning, wherein we analyze both the exponential autocorrelation time and the scaling of variances with bin size. Three-point correlation functions are computed at two source-sink separations $T$ and $T+1$ (see Table 1).

Operators for currents on the lattice are related to those in the continuum according to $\mathcal{J} \doteq Z_{J} J$. We use a mostly nonperturbative matching,

$$
Z_{J}=\rho_{J} \sqrt{Z_{V_{b b}^{4}} Z_{V_{q q}^{4}}},
$$

where $q=l, s$ for $P=\pi, K$, both square-root factors are computed nonperturbatively, and $\rho_{J}$ are computed perturbatively. The one-loop-corrected $\rho_{J}$ are not yet available in the literature. They will be used to introduce a blinding procedure to our analysis, as in Refs. $[12,13]$.

\section{Analysis}

We extract the masses and energies of mesons on the lattice by fitting the two-point correlation functions to these forms:

$$
\begin{aligned}
& C_{2}^{B}(t ; \mathbf{0})=\sum_{n=0}^{2 N-1}(-1)^{n(t+1)} \frac{\left|Z_{B}^{(n)}\right|^{2}}{2 M_{B}^{(n)}}\left[e^{-M_{B}^{(n)} t}+e^{-M_{B}^{(n)}\left(N_{t}-t\right)}\right], \\
& C_{2}^{P}(t ; \boldsymbol{k})=\sum_{n=0}^{2 N-1}(-1)^{n(t+1)} \frac{\left|Z_{P}^{(n)}\right|^{2}}{2 E_{P}^{(n)}}\left[e^{-E_{P}^{(n)} t}+e^{-E_{P}^{(n)}\left(N_{t}-t\right)}\right],
\end{aligned}
$$

where the overlap amplitudes are $Z_{B}^{(n)}=\left\langle 0\left|O_{B}\right| B^{(n)}\right\rangle$ and $Z_{P}^{(n)}=\left\langle 0\left|O_{P}\right| P^{(n)}\right\rangle$. We use $N=3$ in all cases except for the zero-momentum pion, which does not contain states with opposite parity. We loosely constrain the priors for amplitudes and energies according to the procedures outlined in Ref. [14]. For the fit ranges $\left[t_{\min }, t_{\max }\right]$, which are listed in Table 2, we choose $t_{\min }$ in physical units to be consistent for corresponding mesons on different ensembles, as well as to maximize goodness of fit. We choose $t_{\max }$ by requiring that the fractional errors in the two-point correlators remain below $5 \%$. Fit results are confirmed to be stable under variations in $t_{\min }, t_{\max }, N$, and prior widths.

Form factors may be extracted from fits to three-point correlation functions with these forms:

$$
C_{3}^{\mu(v)}(t, T ; \boldsymbol{k})=\sum_{m, n=0}^{2 N-1}(-1)^{m(t+1)}(-1)^{n(T-t-1)} \frac{Z_{P}^{(m)}}{E_{P}^{(m)}} A_{m n}^{\mu(v)} \frac{Z_{B}^{\dagger(n)}}{M_{B}^{(n)}} e^{-E_{P}^{(m)} t} e^{-M_{B}^{(n)}(T-t)},
$$



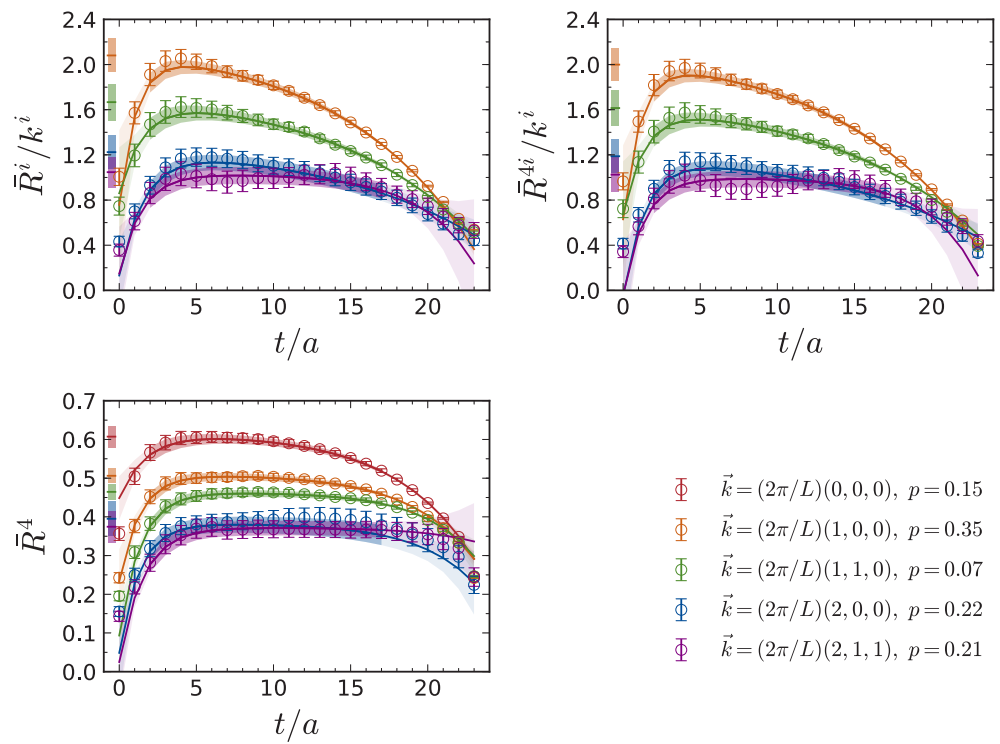

$\begin{array}{ll}\Phi & \vec{k}=(2 \pi / L)(0,0,0), p=0.15 \\ \Phi & \vec{k}=(2 \pi / L)(1,0,0), p=0.35 \\ \Phi & \vec{k}=(2 \pi / L)(1,1,0), p=0.07 \\ \Phi & \vec{k}=(2 \pi / L)(2,0,0), p=0.22 \\ \Phi & \vec{k}=(2 \pi / L)(2,1,1), p=0.21\end{array}$

Figure 2. Form factor ratios $\bar{R}$, fits, and unrenormalized results for $f_{\perp}$ (top left panel), $f_{T}$ (top right panel), and $f_{\|}$ (bottom left panel) in $B \rightarrow \pi$ at $a \simeq 0.088 \mathrm{fm}$ with $m_{l} / m_{s}=$ physical. Fit bands are darker in the fit range, which is identical for all momenta and extends beyond that of the direct fits in both directions. Form-factor results (without $Z_{J}$ ) are indicated to the left of $t / a=0$; they are proportional to the green symbols in the leftmost column of Figure 3.

where $A_{m n}^{\mu(v)}=\left\langle P^{(m)}\left|J^{\mu(v)}\right| B^{(n)}\right\rangle$. Fitting directly to Eq. (4.3) yields the form factors as follows:

$$
\begin{aligned}
f_{\perp}\left(E_{P}\right) & =Z_{V^{i}} \frac{A_{00}^{i}(\boldsymbol{k})}{k^{i}}, \\
f_{\|}\left(E_{P}\right) & =Z_{V^{4}} A_{00}^{4}(\boldsymbol{k}), \\
f_{T}\left(E_{P}\right) & =Z_{T^{4 i}} \frac{M_{B}+M_{P}}{\sqrt{2 M_{B}}} \frac{A_{00}^{4 i}(\boldsymbol{k})}{k^{i}},
\end{aligned}
$$

where the current-renormalization factors $Z_{J}$ are defined in Eq. (3.4). These fits are applied over ranges of $\left[t_{\min }^{P}, T-t_{\min }^{B}\right]$ with loosely constrained fit priors. Alternatively, the form factors may be obtained by computing ratios of three- to two-point correlators, as introduced in Ref. [15]. For the form factors presented in this report, we employ the latter procedure, as exemplified in Figure 2 . The preliminary form factors for $B \rightarrow \pi, B_{s} \rightarrow K$, and $B \rightarrow K$ are shown in Figure 3, where they include only the nonperturbative factors in $Z_{J}$. The perturbative factors $\rho_{J}$ will be blinded $[12,13]$ once they are available. This will reduce subjective bias in our analysis because the $\rho_{J}$ will be unblinded only after finalizing our results.

Our analysis will proceed similarly to Refs. [12, 13, 15]. We will perform a combined chiralcontinuum extrapolation of the form factors. Since the sea quarks are at physical mass in this work, chiral perturbation theory will be used as an interpolation to correct for slight mistunings. We do not expect $b$-quark mistuning to be significant because the parameters are determined with greater precision in this work. Next, we will construct a complete error budget, including uncertainties from 

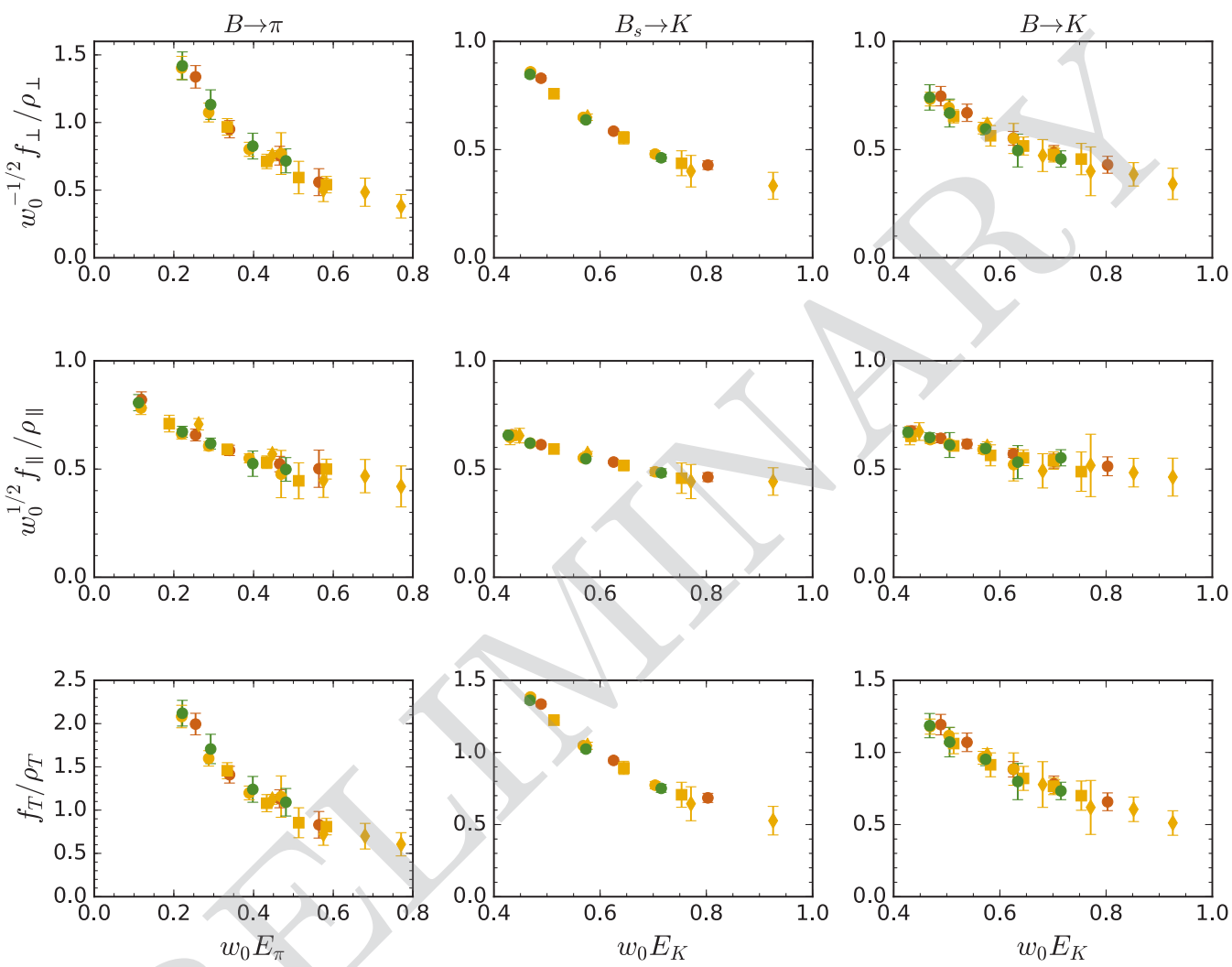

Figure 3. Preliminary results for form factors $f_{\perp}$ (top), $f_{\|}$(middle), and $f_{T}$ (bottom), in $B \rightarrow \pi$ (left), $B_{s} \rightarrow K$ (center), and $B \rightarrow K$ (right), without $\rho_{J}$.

scale, dynamical quark masses, and current renormalization, as well as effects from finite volume and the discretization of quarks and gluons. Lastly, we will extrapolate to the full kinematic range accessible in experiment by applying the model-independent $z$ expansion $[16,17]$. It is then that the form factors will be unblinded and direct comparisons with experiment can be made.

\section{Summary and outlook}

We have presented preliminary results for the lattice-QCD calculation of the form factors $f_{\perp}, f_{\|}$, and $f_{T}$ in the charged-current decays $B_{(s)} \rightarrow \pi(K) \ell v_{\ell}$ and neutral-current decays $B \rightarrow \pi(K) \ell^{+} \ell^{-}$. These 
calculations use HISQ ensembles from MILC, which include physical quark masses. We expect similar statistics and discretization errors to previous asqtad analyses [12,13] for ensembles at similar lattice spacings. The main improvement in this work is the removal of the errors due to the chiral extrapolation. Further improvement will be necessary, both in the statistics for the nonperturbative renormalization factors and in the availability of ensembles at finer lattice spacings.

Our analysis is meant to confront experiment in two ways. First, we will determine $\left|V_{u b}\right|$ by combining with experimental results for the charged-current decays, which will help to address the tension between inclusive and exclusive determinations. Second, we will test for new physics by comparing with experimental results for the neutral-current decays (e.g., branching fractions in $B \rightarrow$ $K \mu^{+} \mu^{-}$), which will help to address the tension between theory and experiment.

\section{Acknowledgments}

This work was supported in part by the U.S. Department of Energy under grants No. DE-AC0506OR23177 (C.B.), No. DE-SC0010120 (S.G.), No. DE-SC0015655 (A.X.K., Z.G.), No. DESC0010113 (Y.M.), No. DE-FG02-13ER41976 (D.T.); by the U.S. National Science Foundation under grants PHY12-12389 (Y.L.), PHY14-14614 (C.D.), and PHY13-16748 and PHY16-20625 (R.S.); by the Fermilab Distinguished Scholars Program (A.X.K.); by German Excellence Initiative and the European Union Seventh Framework Program under grant agreement No. 291763 as well as the European Union's Marie Curie COFUND program (A.S.K.); by the Blue Waters PAID program (Y.L.); and by the Visiting Scholars Program of the Universities Research Association (Z.G., Y.L.).

Computations for this work were carried out with resources provided by the USQCD Collaboration; by the ALCF and NERSC, which are funded by the U.S. Department of Energy; and by NCAR, NCSA, NICS, TACC, and Blue Waters, which are funded through the U.S. National Science Foundation. The Blue Waters sustained-petascale computing project is supported by the National Science Foundation (awards OCI-0725070 and ACI-1238993) and the state of Illinois. Blue Waters is a joint effort of the University of Illinois at Urbana-Champaign and its National Center for Supercomputing Applications. Fermilab is operated by Fermi Research Alliance, LLC under Contract No. DE-AC0207CH11359 with the United States Department of Energy, Office of Science, Office of High Energy Physics. The United States Government retains and the publisher, by accepting the article for publication, acknowledges that the United States Government retains a non-exclusive, paid-up, irrevocable, worldwide license to publish or reproduce the published form of this manuscript, or allow others to do so, for United States Government purposes.

\section{References}

[1] C. Patrignani et al. (Particle Data Group), Chin. Phys. C40, 100001 (2016)

[2] D. Du, A.X. El-Khadra, S. Gottlieb, A.S. Kronfeld, J. Laiho, E. Lunghi, R.S. Van de Water, R. Zhou (Fermilab Lattice), Phys. Rev. D93, 034005 (2016), 1510.02349

[3] B. Aubert et al. (BaBar), Phys. Rev. D73, 012004 (2006), hep-ex/0507054

[4] K. Abe et al. (Belle), Phys. Rev. D71, 072003 (2005), [Erratum: Phys. Rev.D71,079903(2005)], hep-ex/0408111

[5] A. Abulencia et al. (CDF), Phys. Rev. Lett. 97, 242003 (2006), hep-ex/0609040

[6] V.M. Abazov et al. (D0), Phys. Rev. Lett. 110, 011801 (2013), 1207.1769

[7] R. Aaij et al. (LHCb), Eur. Phys. J. C73, 2655 (2013), 1308 . 1302

[8] R. Aaij et al. (LHCb), JHEP 06, 133 (2014), 1403.8044

[9] R. Aaij et al. (LHCb), JHEP 10, 034 (2015), 1509.00414 
[10] S. Borsanyi et al., JHEP 09, 010 (2012), 1203 . 4469

[11] A. Bazavov et al. (MILC), Phys. Rev. D93, 094510 (2016), 1503.02769

[12] J.A. Bailey et al. (Fermilab Lattice, MILC), Phys. Rev. D92, 014024 (2015), 1503.07839

[13] J.A. Bailey et al. (Fermilab Lattice, MILC), Phys. Rev. D93, 025026 (2016), 1509.06235

[14] A. Bazavov et al. (Fermilab Lattice, MILC), Phys. Rev. D93, 113016 (2016), 1602.03560

[15] J.A. Bailey et al. (Fermilab Lattice, MILC), Phys. Rev. D79, 054507 (2009), 0811. 3640

[16] C.G. Boyd, B. Grinstein, R.F. Lebed, Phys. Rev. Lett. 74, 4603 (1995), hep-ph/9412324

[17] C. Bourrely, I. Caprini, L. Lellouch, Phys. Rev. D79, 013008 (2009), [Erratum: Phys. Rev.D82,099902(2010)], 0807.2722 